\title{
Supply Chain Logistics in Multi-Level Just-in-Time Production Sequencing Problems
}

\author{
Gyan Bahadur Thapa ${ }^{1}$, Sergei Silvestrov ${ }^{2}$ \\ ${ }^{1}$ Pulchowk Campus, Institute of Engineering, Tribhuvan University, P. O. Box 19758, Kathmandu, Nepal \\ ${ }^{2}$ Division of Applied Mathematics, Mälardalen University, Box 883, 72123 Vasteras, Sweden \\ ${ }^{a}$ Corresponding author: thapagbt@ioe.edu.np
}

\begin{abstract}
The multi-level just-in-time sequencing problem is one of the challenging research areas in supply chain management. In this paper, we present brief review and some recent research developments of just-in-time production systems together with supply chain logistics. Observing production flows and supply chain synchronization in production process, we present the mathematical models of just-in-time (JIT) sequencing problem in multi-level and single-level as nonlinear integer programming in terms of discrepancy functions under the specified constraints. Discrete apportionment approach is briefly reported as an efficient frontier for single-level.
\end{abstract}

Keywords Just-in-time, JIT sequencing, flows, supply chain, logistics

\section{Introduction}

In today's global and dynamic production environment, the demand for high quality goods in minimum cost is more prevalent which needs a well-managed supply chain and logistics systems. Generating an optimal production schedule for an assembly line to balance the overall supply chain under a variety of practical constraints is a difficult task. Many companies are trying to make their production systems more flexible or adaptable with change to respond the diverse customer demands. One of the most significant concepts of production in past decades has been JIT production system (JITPS) originated in Toyota, Japan around 1970s [16]. JITPS is a management philosophy and a technique based upon the idea that no activity should take place until there is a need for it; that is, no products should be made or ordered if there is no demand. On top of this, it is a pull production system where demand pulls goods towards the market. JIT production logistics forms a specific part of the supply chain that deals with the planning and controlling of materials and information flows throughout the production and distribution systems of manufacturing companies.

The JIT philosophy focuses on waste elimination and inventory reduction. This requires producing and transporting the right items in the right quantities at the right time with desired quality [14]. The JIT logistics is performed to optimize some sort of given performance measures, for example minimizing total operating costs, and to satisfy a given set of constraints, 
for example budget constraints. The crux of the JITPS is to produce goods in mixed-model production sequence to synchronize with the different demands of the customers with minimal inventories and shortages. The sequenced delivery of the materials and products throughout the supply chain of companies is the ultimate realization of JIT principles - zero inventories, zero defects, zero waste. Sequencing problem is a problem that finds a permutation or an ordering of a finite collection of jobs or products that satisfies certain conditions, such as precedence constraints, integrality constraints, monotonic constraints [6]. Some of the benefits of JITPS are reduced inventory, improved quality, shorter lead times, lower production costs, increased productivity, increased workforce flexibility and greater output volume flexibility.

The two types of JITSPs are studied in the literature: single-level [7] and multi-level [9, 12]. The single-level problem minimizes the variations in the product rates at which different products are produced on the production line. The multi-level problem is to minimize the variations in demand rates for outputs of supplying processes. Moreover, the objective of both the problems is to minimize the discrepancies between ideal and actual productions. To attain this goal, JITPS penalizes both earliness and tardiness of the operations which has spawned a rapidly developing line of research in scheduling theory. The time needed to switch from one product to another is assumed to be negligible.

The rest of the paper is planned as follows: Section 2 describes the logistics flows in supply chain and Section 3 presents the supply chain synchronization. The mathematical models of multi-level and single-level JITSPs are given in Sections 4 and 5 respectively. Section 6 presents an efficient frontier for the single-level JIT sequencing problem, and finally Section 7 concludes the paper.

\section{Production Flows in Supply Chain}

Production logistics is the movement of materials, data and products into, through and out of manufacturing companies. Inbound logistics covers the movement of materials received from initial suppliers, and outbound logistics refers the physical distribution of finished goods to the customers. In general, there exist four types of flows between suppliers and customers in their production and distribution networks as shown in Fig. 1 below [17]. We describe them briefly as follows: (a) Goods flows are the external logistics flows where transportation is predominant, and storage or value added logistics may occur on the way. The goods flow may be part of physical distribution for suppliers or part of physical collection for the customers. (b) Transaction flows are generalizations of markets and sells, which are commercial distribution in view of suppliers and commercial supply in view of customers. (c) Money flows are mainly payments for the goods received. (d) Information flows are two way flows and usually there are a number of them related to each goods flow; closely related to transaction and money flows often transformed into information flows. 


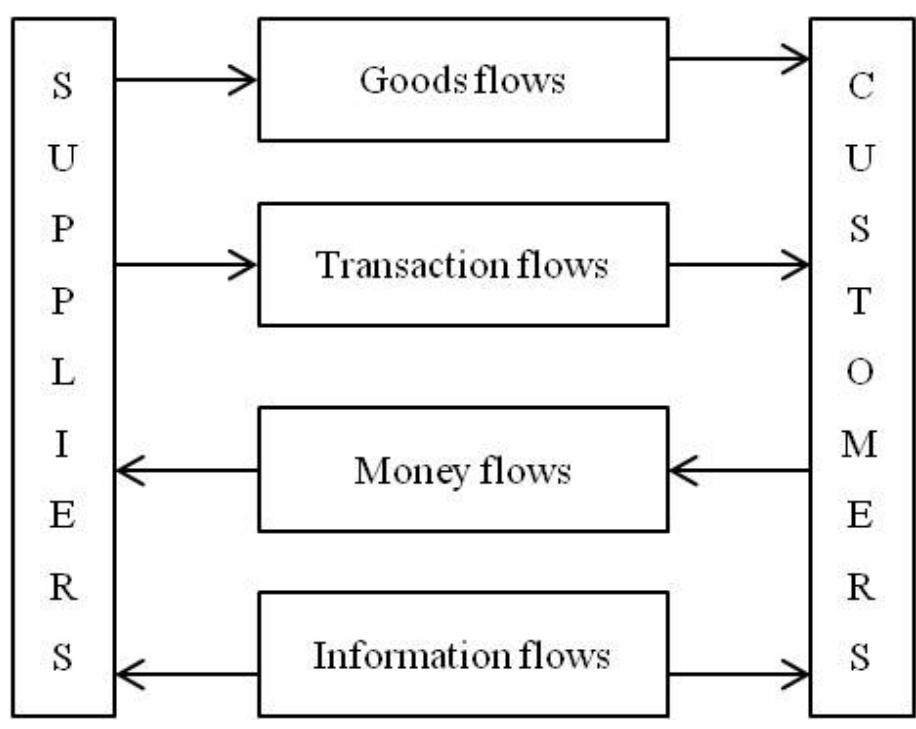

Fig. 1. Production and distribution network flows

Information may be transformed in time, space and in form to optimize the operations [20, 21]. Modern production management is oriented towards automation and computer-integrated manufacturing, heavily relying on data flows as a controlled flow of materials producing information along with products and by-products.

\section{Supply Chain Synchronization}

Production and logistics are like the two faces of a coin. The integration of overall production and distribution processes within and among companies is being the most fundamental trend in production management. A synchronized view of these processes includes all traditional areas of supplier-buyer relationships coping with the production of goods and their distribution as well [19]. The supply chain system consists of three major parts: procurement, production and distribution. The multi-level JIT sequencing problem (JITSP) consists of several levels in the production supply chain: raw materials $\rightarrow$ components $\rightarrow$ subassemblies $\rightarrow$ final products $\rightarrow$ distribution centres $\rightarrow$ retailers $\rightarrow$ final customers. In this chain, multiple copies of different models are produced at final assembly level, which is interlinked with several upstream production levels where raw materials are procured, stored and fabricated to produce the final products and with several downstream distribution levels where final products are stored and distributed to the retailers and then to the customers. The whole body of supply chain consists of inbound logistics along the production levels and outbound logistics along the distribution levels. The Fig. 2(a), Fig. 2(b) and Fig. 2(c) best illustrate this situation as synchronized view of seven levels of production and supply chain networks. 


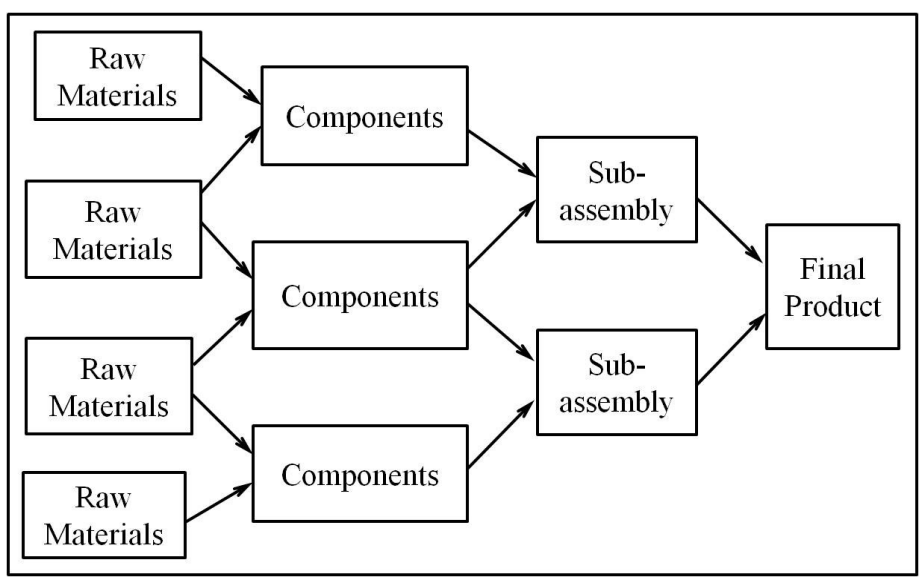

Fig. 2(a). Production levels



Fig. 2(b). Distribution levels

There can occur several sublevels in between any two production levels and hence the mathematical model of the multi-level JITSP contains $L$ levels, where $l=1,2, \cdots, L$. The aim of supply chain synchronization is to coordinate the flow of materials, products, and information between supply chain partners to reduce the overproduction, underproduction and duplication.

To realize the best quality production and timely distribution to the customer in a rapidly changing scenario, it is essential to create a cross-docking environment throughout whole supply chain that is capable to address the diversified demands. It is the movement of products directly from receiving dock to shipping dock with minimum dwell time in between. By arranging immediate cross-docks of incoming products, retailers are able to reduce in-transit time for their incoming products. This is relatively new logistics technique used in the retail and trucking industries with operations seeking to move materials from inbound to outbound locations as quickly as possible, that requires good information systems and close synchronization of all 
inbound and outbound shipments [18]. The implementation of cross-docking in food products distribution can lead to the significant cost advantages and savings, particularly for small orders and frequent deliveries, e.g., perishable products. The cross-docking logistics problem is formulated as truck sequencing problem $[2,18]$.



Fig. 2(c). Supply chain synchronization

\section{Multi-Level Product Sequencing Problem}

Multi-level JITSP consists of $L$ production levels such that $l=1,2, \cdots, L$ with the first product level 1 . The number of different part types and the demand of item $i$ in level $l$ are denoted by $n_{l}$ and $d_{i l}$ respectively, $i=1,2, \cdots, n_{l}$. The number of total units of item $i$ at level $l$ required to produce one unit of the product $p$ is denoted by $t_{i l p}$ such that $d_{i l}=\sum_{p=1}^{n_{1}} t_{i l p} d_{p 1}$ is the dependent demand for item $i$ at level $l$ determined by the final product demands $d_{p 1}=1,2, \cdots, n_{1}$ and $l=1,2, \cdots, L$. Note that $t_{i l p}=1$ if $i=p$ and 0 if otherwise. Finally, $D_{l}=\sum_{i=1}^{n_{1}} d_{i l}$ denotes the total demand at level $l$, and the ratio $r_{i l}=\frac{d_{i l}}{D_{l}}$ gives the demand rate for item $i$ of level $l$ such that $\sum_{i=1}^{n_{l}} r_{i l}=1$ at each level $l=1,2, \cdots, L$. The production schedule at level 1 consists of $D_{1}$ stages in total and at each stage a single unit of an end-product can be processed. An item is at stage $k, k=1, \cdots, D_{1}$, if $k$ units of product is produced at level 1 and there will be $k$ complete units of products $p$ at level 1 during the first $k$ time units. 
Let $x_{i l k}$ be the necessary quantity of item $i$ produced at level $l$ during the time units 1 through $k$ and $y_{l k}=\sum_{i=1}^{n_{l}} x_{i l k}$ be the cumulative quantity of item $i$ produced at level $l$ during the same time units such that $y_{1 k}=\sum_{i=1}^{n_{1}} x_{i 1 k}=k$. Due to pull nature of JITPS, particular combination of the highest level products produced during $k$ time units determines the necessary cumulative production at every other level. Thus, the required cumulative production for item $i$ at level $l$ with $l \geq 2$ through $k$ time units is given by $x_{i l k}=\sum_{p=1}^{n_{1}} t_{i l p} x_{p 1 k}$. For a convex penalty function $F_{i}, i=1,2, \cdots, n_{l}$ with minimum 0 at 0 , the maximum deviation and the sum deviation multilevel JITSPs are mathematically formulated to minimize the objectives $Z_{\max }$ and $Z_{\text {sum }}$ as follows $[9,10,20]:$

$$
\begin{gathered}
Z_{\max }=\min \max _{i, l, k} F_{i}\left(x_{i l k}-y_{l k} r_{i l}\right) \\
Z_{\text {sum }}=\min \sum_{k=1}^{D_{1}} \sum_{l=1}^{L} \sum_{i=1}^{n_{l}} F_{i}\left(x_{i l k}-y_{l k} r_{i l}\right)
\end{gathered}
$$

subject to

$$
\begin{aligned}
& x_{i l k}=\sum_{p=1}^{n_{1}} t_{i l p} x_{p 1 k}, i=1,2, \cdots, n_{l} ; l=1,2, \cdots, L ; k=1,2, \cdots, D_{1} \\
& y_{l k}=\sum_{i=1}^{n_{l}} x_{i l k}, \quad l=2,3, \cdots, L ; k=1,2, \cdots, D_{1} \\
& y_{1 k}=\sum_{p=1}^{n_{1}} x_{p 1 k}=k, \quad k=1,2, \cdots, D_{1} \\
& x_{p 1 k} \geq x_{p 1(k-1)}, \quad p=1,2, \cdots, n_{1} ; k=1,2, \cdots, D_{1} \\
& x_{p 1 D_{1}}=d_{p 1}, x_{p 10}=0, \quad p=1,2, \cdots, n_{1} \\
& x_{i l k} \geq 0, \text { integer and } i, l, k \text { as above }
\end{aligned}
$$

The constraint (3) ensures that the necessary cumulative production of part $i$ of level $l$ by the end of time unit $k$ is determined explicitly by the quantity of products produced at level 1 . Constraints (4) and (5) show the total cumulative production of level $l$ and level 1 respectively during the time slots 1 through $k$. Constraint (6) ensures that the total production of every product over $k$ time units is a non-decreasing function of $k$. Constraint (7) guarantees that the demands for each product are met exactly, and (8) is the integral constraint. The constraints (5), (6), (8) jointly ensure that exactly one unit of a product is scheduled during one time unit in the product level. This is an integer programming problem targeting to find the best production 
sequence in mixed-model that minimizes one of the objectives (1) or (2) under the constraints (3) to (8). This model is assumed to be non-preemptive (i.e., once commenced production at level 1 must be completed prior to switch into another unit).

The particular cases of the min-max objective (1) and the min-sum objective (2) are studied in terms of absolute and squared deviations, and the weighted cases with NP-hard results are also discussed in $[5,16]$. The objective (1) aims to find a smooth schedule in every time period for every output. It is the basic concept underlying Toyota's sequencing algorithm [11]. The objective (2) seeks optimal schedules that may have relatively large deviation in a single period or for a certain output while having the lowest possible total deviation. This problem is NP-hard in general. However some heuristics [11, 20]; the dynamic programming [9] and pegging assumption [12] exist for heuristic and suboptimal solutions.

\section{Single-Level Sequencing Problems}

Suppose there are $n$ products to be produced within the specified time horizon with the integer demands $d_{1}, d_{2}, \ldots \ldots, d_{n}$ such that $\sum_{i=1}^{n} d_{i}=D$. If $r_{i}=\frac{d_{i}}{D}$ is the ideal production rate for the parts of type $i$ such that $\sum_{i=1}^{n} r_{i}=1$, then the scheduling goal for the assembly line is to maintain the total cumulative production of product $i$ to the total production as close to $r_{i}$ as possible. This means exactly $k r_{i}$ units of product $i$ should be produced in the first $k$ time period, which is the ideal production where $k=1,2, \cdots, D$.

Let $x_{i k}, i=1,2, \cdots, n ; k=1,2, \cdots, D$, be the actual cumulative production of product $i$ in the time period 1 through $k$. For a convex symmetric penalty function $F_{i}, i=1,2, \cdots, n$ with minimum $F_{i}(0)=0$; the maximum deviation and the sum deviation JITSPs are formulated to minimize $F_{\max }$ and $F_{\text {sum }}$ as follows $[4,5,8,14,15]$ :

$$
\begin{gathered}
F_{\max }=\min \max _{i, k} f_{i}\left(x_{i k}-k r_{i}\right) \\
F_{\text {sum }}=\min \sum_{i=1}^{n} \sum_{k=1}^{D} f_{i}\left(x_{i k}-k r_{i}\right)
\end{gathered}
$$

subject to

$$
\begin{aligned}
& \sum_{i=1}^{n} x_{i k}=k, k=1,2, \cdots, D \\
& x_{i(k-1)} \leq x_{i k}, i=1,2, \cdots, n ; k=1,2, \cdots, D \\
& x_{i D}=d_{i}, i=1,2, \cdots, n \\
& x_{i k} \text { is a non-negative integer }
\end{aligned}
$$


The constraint (11) ensures that exactly $k$ units are scheduled in periods 1 to $k$, and constraint (12) represents the monotone condition guarantying total production of every product over $k$ is non-decreasing. The constraint (13) ensures that production requirements are met for each product. These constraints indicate that exactly one product is produced during each stage. It is an integer programming problem with cardinality, monotonicity and integrality constraints. The perfect matching [13] for maximum deviation case, and assignment approach [8] for sum deviation case are available. The stronger bottleneck and some algebraic properties of maximum deviation with some conjectures are provided [3]. An efficient frontier is given for sum deviation case via discrete apportionment [4].

Example: We explain how mixed-model production sequences are generated. Suppose the demands of three models $a, b$ and $c$ of a base product be $d_{1}=7, d_{2}=5$ and $d_{3}=2$ units respectively such that the total demand is $D=14$. One of the production sequences based on

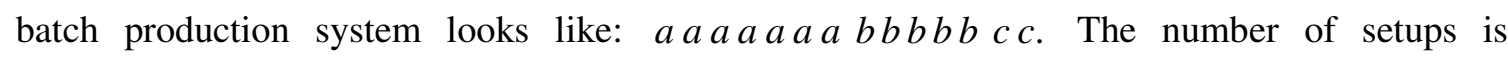
minimized in this sequence. But production is unsynchronized with demand which generates large inventories and customers have to wait to get their choices. To avoid this defect, the companies have to inter-mix the models to exploit the benefits of mixed-model sequence. Here, we observe the four mixed-model sequences as follows:

abababcababaca; bababacbabaaca; ccaabababababa ;

$a b a a b a c b a b b c a a$. The number of possible sequences associated with this example in total are $\frac{D !}{d_{1} ! d_{2} ! d_{3} !}=\frac{14 !}{7 ! 5 ! 2 !}=72,072$. In mixed-model production system, each product is produced concurrently with respect to the demand, small batches of each product are produced and hence there occurs small inventories. The detail explanation is found in $[4,16]$.

\section{Discrete Apportionment: an Efficient Frontier}

The sum deviation JITSP is simultaneously handled via discrete apportionment, which is a fair division problem aiming to allocate integer seats to sates or parties according to population size or votes [4]. The crux of the problem is to minimize the deviation between ideal and actual apportionments. Given house size $h$, let $s$ states are to get integer seats $a_{i}, i=1, \cdots, s$ such that $\sum_{i=1}^{s} a_{i}=h$ with total population $\sum_{i=1}^{s} p_{i}=p$. The ideal quota is calculated by $q_{i}=\frac{p_{i} h}{p}$ which is not necessarily an integer. The generic problem is to minimize $\sum_{i=1}^{s}\left(a_{i}-q_{i}\right)^{2}$ or $\sum_{i=1}^{s}\left|a_{i}-q_{i}\right|$ subject to $\sum_{i=1}^{s} a_{i}=h$ and $a_{i} \in Z^{+}$. One can assign either $a$ or $a+1$ seats to a state. Among various apportionment methods [1], divisor methods are dealt and mean-based divisor methods are established providing stronger bounds [4]. The sum deviation JIT objectives are compared with the apportionment objective functions in terms of local and global deviations. 
and sufficient conditions are established with same set of optimal solutions, i.e., the sum deviation JITSP objective and apportionment objective are equivalent. The complexities of the problems are also equivalent being $O(n D)$ and $O(s h)$ respectively. The ideal case of JITSP is $x_{i k}-k r_{i}=0$, which is very rare to attain. So we need to find the smallest possible upper bound that minimizes the overproduction and underproduction. 1 and $1-\frac{1}{D}$ are the two upper bounds studied so far. Our key idea is to compute a stronger bound setting $a=0$ and $a+1=0+1=1$ from the mean-based partition given to be $\frac{1}{5}\left(0+0+0+\frac{1}{2}+1\right)=0.3$ which works for both problems reducing the deviations significantly. Also, product to product rate variation problem is shown to be equivalent with pair-wise apportionment problem with their respective relative differences [4].

\section{Conclusion}

The mixed-model JITSP has been widely studied with various mathematical aspects and solution approaches. But it is still challenging area due to its interesting base model of theoretical value and wide real-world applications. Single-level problem is solvable in pseudo-polynomial time, but multi-level one is NP-hard. An efficient frontier via discrete apportionment is presented for single-level. The simultaneous study of production and logistics is still a challenging area having many research issues. Our further work will be focused on synchronized study of production and logistics to balance overall supply chain.

\section{References}

[1] Balinski M and Young HP (2001), Fair representation: meeting the ideal of one man, one vote. 2nd edn, Brookings Institution Press, Washington D.C.

[2] Boysen N, Fliedner M and Scholl A (2010), Scheduling inbound and outbound trucks at crossdocking terminals. Operations Research Spectrum 32 : 135-161.

[3] Brauner N and Crama Y (2004), The maximum deviation just-in-time scheduling problem. Discrete Applied Mathematics 134 : 25-50.

[4] Dhamala TN, Thapa GB and Yu H (2012), An efficient frontier for sum deviation just-in-time sequencing problem in mixed-model systems via apportionment. International Journal of Automation and Computing 9(1) : 87-97.

[5] Dhamala TN and Kubiak W (2005), A brief survey of just-in-time sequencing for mixedmodel systems. International Journal of Operations Research 2(2) : 38-47.

[6] Khashouie GM (2003), Sequencing mixed-model assembly lines in just-in-time production systems. Dissertation, Brunel University, UK.

[7] Kubiak W (1993), Minimizing variation of production rates in just-in-time systems: A survey. European Journal of Operational Research 66 : 259-271.

[8] Kubiak W and Sethi S (1994), Optimal just-in-time schedules for flexible transfer lines. The International Journal of Flexible Manufacturing Systems 6 : 137-154. 
[9] Kubiak W, Steiner G and Yeomans JS (1997), Optimal level schedules for mixed-model multi-level just-in-time assembly systems. Annals of Operations Research 69 : 241-259.

[10] Miltenburg J and Sinnamon G (1989), Scheduling mixed-model multi-level just-in-time production systems. International Journal of Production Research 27(9): 1487-1509.

[11] Monden Y (1983), Toyota Production System; Practical Approach to Production Management, Industrial Engineering and Management Press, Norcross, GA.

[12] Steiner G and Yeomans JS (1996), Optimal level schedules in mixed-model multi-level justin-time assembly systems with pegging. European Journal of OR 95 : 38-52.

[13] Steiner G and Yeomans J (1993), Level schedules for mixed-model just-in-time processes. Management Science 39(6): 728-735.

[14] Thapa GB and Dhamala TN (2009), Just-in-time sequencing in mixed-model production systems relating with fair representation in apportionment theory. The Nepali Mathematical Sciences Report 29 : 29-68.

[15] Thapa GB and Dhamala TN (2009), A synthetic study to minimize the inequality measures in just- in-time sequencing problem via optimization methods. Proceedings of the 5th Asian Mathematical Conference 2009, Malaysia, III 978-967-5417-54-2, 462-470.

[16] Thapa GB (2012), Characterizations of just-in-time sequencing problems with apportionment and supply chain. Dissertation, Tribhuvan University, Nepal.

[17] Thapa GB (2014), Basics of informed logistics in just-in-time production sequencing and supply chain systems. Journal of Institute of Engineering, 9(1) : 54-64.

[18] Thapa GB, Dhamala TN and Pant SR (2011), Cross-docking operations for supply chain logistics in JIT production systems. Journal of Institute of Engineering, 8(1) : 219-230.

[19] Thapa GB, Dhamala TN and Yu H (2010), Mathematical model of cross-docking operations in supply chain systems under multi-level just-in-time production environment. The Nepali Mathematical Sciences Report 30(1\&2) : 24-35.

[20] Thapa GB (2015), Optimization of just-in-time sequencing problems and supply chain logistics. Dissertation, Division of Applied Mathematics, Malardalen University, Sweden.

[21] Tilanus B (1997), Information systems in logistics and transportation, 2nd edn. Pergamon press. 